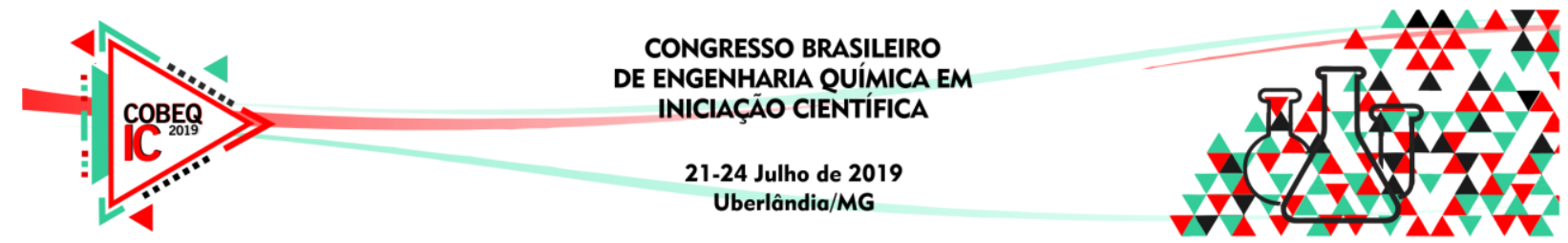

\title{
SIMULAÇÃO DO LIMIAR DE PERCOLAÇÃO DE NANOTUBOS DE CARBONO EM MATRIZ POLIMÉRICA: AVALIAÇÃO TRIDIMENSIONAL DA TORTUOSIDADE DAS NANOCARGAS.
}

\author{
J.S.PEREIRA ${ }^{1}$, P.H.S.L.COELHO ${ }^{1}$, R.P.VIEIRA ${ }^{1}$, N.E.C.L. SOUZA ${ }^{1}$, V.A.D. ARMELLINI ${ }^{2}$ \\ ${ }^{1}$ Universidade Federal do Maranhão, Departamento de Engenharia Química \\ ${ }^{2}$ Faculdade de Campinas, Departamento de Engenharia de Produção
}

E-mail para contato: je.pereira002@gmail.com

\begin{abstract}
RESUMO - Nanocompósitos poliméricos condutores constituídos por nanotubos de carbono apresentam um alto potencial para aplicações. Porém, o alto custo destas cargas influencia na melhor investigação das condições de processo e no estudo da percolação elétrica. Nesta vertente, a modelagem e simulação deste limiar têm um papel importante no desenvolvimento deste campo de estudo e impulsionou a idealização deste trabalho, onde foi realizado um estudo da percolação elétrica de nanocompósitos constituído por nanotubos dispersos em matriz polimérica, simulada através de um programa elaborado pelo método de Monte Carlo e apoiado ao modelo do volume excluído, com objetivo de analisar a tortuosidade geométrica das partículas condutoras em sistemas matriciais bidimensionais e tridimensionais. Após as simulações, notou-se uma diminuição no limiar de percolação com o aumento da razão de aspecto e conforme a partícula se tornava mais tortuosa. Os resultados obtidos para o sistema tridimensional confirmaram sua melhor adequação experimental quanto aos sistemas bidimensionais.
\end{abstract}

\section{INTRODUÇÃO}

Nas últimas décadas, a aplicação de materiais poliméricos se intensificou, sobretudo no ramo da engenharia e tecnologia, por apresentar atributos únicos de grandes vantagens, tais quais a sua fácil produção, processamento e leveza. Entretanto, ao ser comparado com os metais, estes materiais contém uma baixa resistividade, tenacidade e condutividade (HUANG, CHENG, 2017). Dessa forma, a fim melhorar as propriedades dos polímeros, vem se tornando frequente, o estudo de incorporação de cargas tais como nego de fumo, nanotubos de carbono (NTC), grafite, fibras de carbono em matriz polimérica, os chamados compósitos poliméricos condutores (SUN et al., 2009).

Dentre as cargas existentes, a utilização do NTC vem se destacando por conter uma longa estrutura cilíndrica, com alta razão de aspecto ( $r a)$ (SOLEIMANI et al., 2018) e excelentes propriedades mecânicas, elétricas e térmicas (LIU, KUMAR, 2014). Ao dispô-lo na matriz polimérica forma-se uma rede de percolação, ou seja, caminhos que possibilitam o 


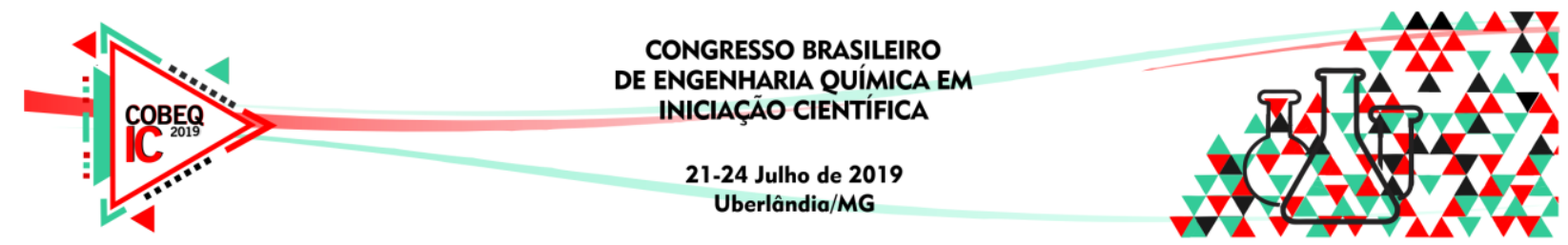

transporte de corrente elétrica, em uma certa concentração crítica, denominada limiar de percolação $\left(\mathrm{p}_{\mathrm{c}}\right)$. O limiar de percolação dos NTC é estudado pelo modelo do volume excluído proposto por Balberg et al., (1984), que considera a sua geometria como bastões ou cilíndricos esféricos. Este modelo consiste, em definir um objeto, no qual seu centro, não se sobrepõe ao centro de um outro objeto semelhante. Através deste modelo, juntamente com simulações associado ao método de Monte Carlo é possível "prever" o valor de $\mathrm{p}_{\mathrm{c}}$ em um sistema, pois os autores consideram o volume excluído $\left(\mathrm{V}_{\mathrm{ex}}\right)$ e o limiar de percolação $\left(\mathrm{p}_{\mathrm{c}}\right)$, grandezas inversamente proporcionais (BALBERG et al., 1984; KUMAR, RAWAL, 2016). De acordo com este modelo, para um sistema em 3D, pode-se definir a concentração crítica de percolação através do volume total excluído das partículas $V_{e x}$ conforme a Equação 1.

$$
\mathrm{p}_{\mathrm{c}}=1-\exp \left(-\frac{\left\langle V_{\text {ex }} V\right\rangle}{\left\langle V_{\mathrm{f}}\right\rangle}\right)=1-\exp \left(-N_{c} V\right)
$$

Muitos estudos sobre a percolação elétrica desses sistemas consideram que as cargas condutoras têm a forma de cilindros ou esferocilindros rígidos e retos (BALBERG et al., 1984; BERHAN, SASTRY, 2007; MUNOZ-SANDOVAL et al., 2017). Tal aproximação, apesar de ser relativamente boa para a maioria dos sistemas, descreve mal a realidade dos nanocompósitos, visto que a realidade estrutural microscópica destas cargas, revela um comportamento ondular emaranhado. Portanto, modelos de percolação que incluem a chamada tortuosidade, são necessários para melhor representação destes sistemas.

O objetivo deste estudo foi determinar, em duas dimensões (2D) e em três dimensões (3D), o limiar de percolação elétrico de partículas de NTC em matriz polimérica, simulando o efeito de diferentes tortuosidades das nanocargas na concentração crítica por meio do método numerico de Monte Carlo apoiado ao modelo do volume excluído.

\section{METODOLOGIA}

Os algoritmos utilizados para a montagem das matrizes e realização das simulações foram desenvolvidos em linguagem FORTRAN 95 (Force 2.0.9). O simulador gera matrizes bidimensionais quadradas com 1000 elementos de lado e matrizes tridimensionais cúbicas, com 750 elementos de aresta. Os pontos destas matrizes são definidos, inicialmente, com o valor 0 , e representam o material polimérico isolante.

A fração volumétrica das partículas de NTC presentes na matriz é determinada no início do programa pelo próprio usuário, assim como a $r a$. Para inserção da $r a$ no simulador, foi definido como unitário o diâmetro da fibra, para que o valor inserido na programação fosse apenas o comprimento do tubo.

Partindo do valor da fração volumétrica informada, os programas calculam a quantidade de partículas que devem ser geradas para atender à porcentagem de material condutor fornecida. Desta forma, calculamos via número de NTC a fração volumétrica desejada pelo usuário ( $\left.V_{\mathrm{NTC}}\right)$, para 2D, Equação 2, e 3D, Equação 3:

$$
\mathrm{N}_{\mathrm{NTC}_{x} 2 \mathrm{D}}=\frac{10^{6} \cdot \mathrm{V}_{\mathrm{NTC}}}{\mathrm{ra}}
$$




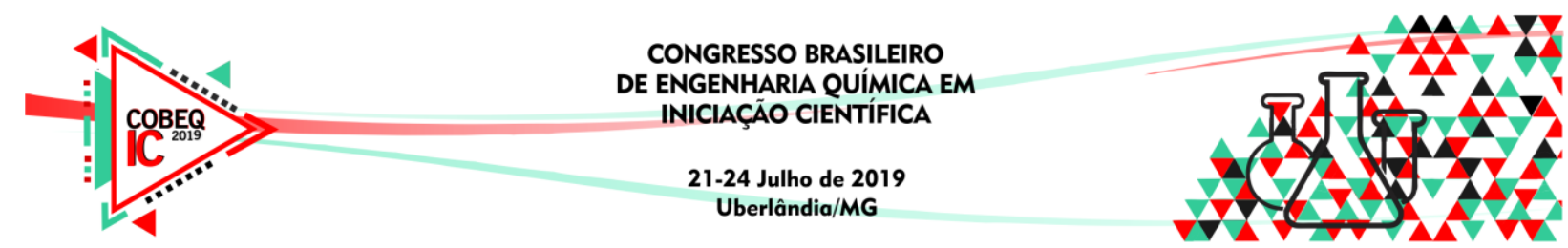

$$
\mathrm{N}_{\mathrm{NTC}, 3 \mathrm{D}}=\frac{750^{3} \cdot \frac{\mathrm{V}_{\mathrm{NTC}}}{100}+1}{\mathrm{ra}}
$$

Os elementos iniciais, a partir dos quais essas partículas são construídas, são escolhidos aleatoriamente pelo programa através do Método de Monte Carlo, por meio da geração de 2 posições aleatórias para matriz 2D (coordenadas x e y) e 3 números aleatórios para a matriz 3D (coordenadas nos eixos $\mathrm{x}, \mathrm{y}$ e $\mathrm{z}$ ). Estes pontos iniciais, que anteriormente eram definidos como 0 , recebem um novo valor 1 , representando o material condutor. A partir destes pontos, as partículas são geradas de acordo com suas respectivas geometrias, incluindo o conceito de tortuosidade das cargas.

Após a geração destas partículas condutoras e a confirmação da fração ocupada pelo material condutor, é realizada uma nova varredura partindo da face superior da matriz à face inferior, verificando se há caminhos formados entre os pontos que permitam a condução da corrente elétrica ao longo de toda a matriz, isto é, se há um caminho contínuo formado pelos valores de partículas. A fim de confirmar um valor ótimo para o limiar de percolação, 100 matrizes são geradas em cada simulação, e o valor de $p_{c}$ é definido como a concentração de partículas de NTC, que percola pelo menos 50\% destas matrizes. Este valor é chamado de possibilidade de conexão (PC) (CHEN et al., 2014).

\section{RESULTADOS E DISCUSSÕES}

\subsection{Simulação dos sistemas bidimensionais}

Foram testadas 3 geometrias tortuosas, com 4 valores de razão de aspecto $(500,1000$, 2000 e 4000). A Figura 1 mostra as geometrias 2D tortuosas denominadas A, B e C, enquanto que a geometria linear discretizada recebeu a denominação D.

Figura 1 - Partículas bidimensionais com diferentes geometrias e $r a=40$.
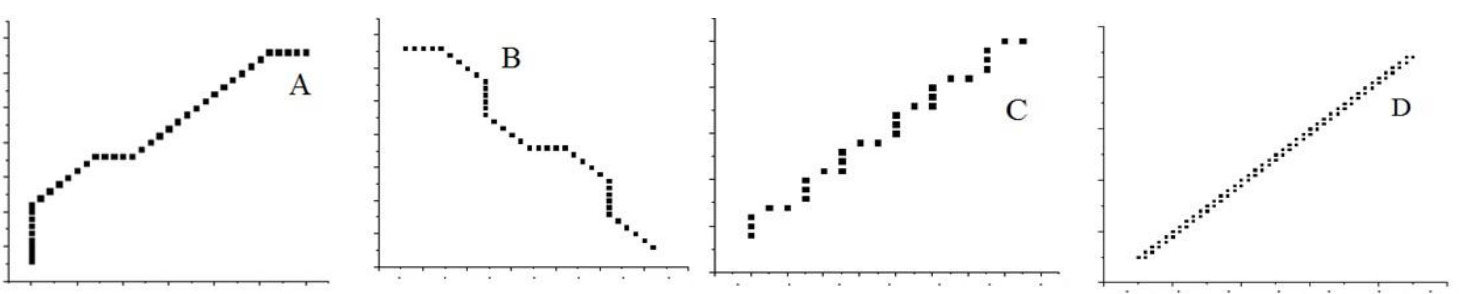

A Figura 2 revela a tendência esperada, onde quanto maior a $r a$, menor o valor de $\mathrm{p}_{\mathrm{c}}$. Comparando-se a geometria D com os autores Coelho et al. (2017), que utlizaram a mesma discretização, pode-se observar que os resultados da simulação, para os mesmos métodos e modelos aplicados, mostraram valores até $50 \%$ inferiores, se adequando melhor ao modelo do volume excluído de Balberg et al (1984), porém, com valores de $\mathrm{p}_{\mathrm{c}}$ acima do que seria a real condição, 3D. Esta dificuldade de adequação ao modelo para o sistema 2D foi suavizada pela inclusão da tortuosidade, que influenciou positivamente na diminuição do limiar. Para estas geometrias tortuosas a menor concentração encontrada, $\mathrm{p}_{\mathrm{c}}=0,15 \%$ vol. de NTC, foi para a Geometria A, com razão de aspecto 4000, e com tortuosidade mais evidente que nas 


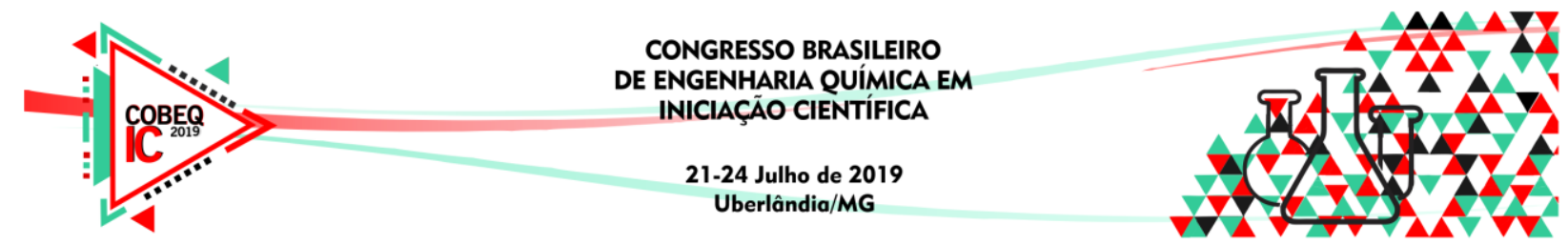

outras duas simuladas. Acredita-se que devido à sua geometria, a probabilidade de contato com as partículas vizinhas foi facilitada pela maior expansividade da partícula, obtendo assim um maior alcance geográfico. Comparando-se os valores de $\mathrm{p}_{\mathrm{c}}$ simulados em 2D com resultados experimentais da literatura, pode-se observar algumas concordâncias como, por exemplo, os limiares pertencentes a $r a$ de 1000, que se encontra em uma faixa de 0,6 a 1,0\%, sendo o melhor valor equivalente a $0,675 \%$ vol. (geometria A), estão condizentes com os valores encontrados por Bug et al. (2007) o qual obteve a menor $\mathrm{ra}$ em torno de $0,6 \%$ vol..

Figura 2 - Limiar de percolação em função da ra.para as diferentes geometrias.

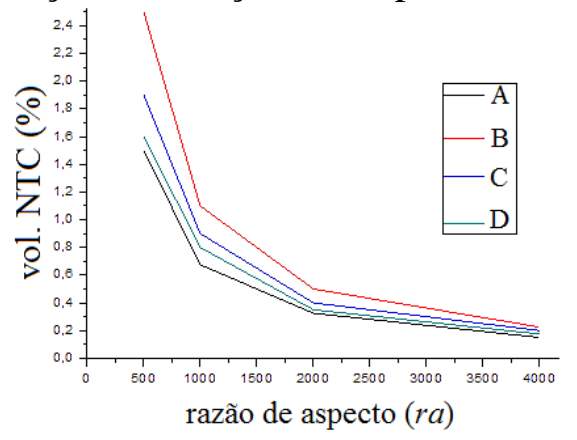

\subsection{Simulação dos sistemas tridimensionais}

Foram simuladas duas geometrias: tortuosa, Figura 3a; e discretizada, Figura 3b, ambas analisa para 4 valores de $r a(50,100,150,250)$.

Figura 3 - Partículas tridimensionais com diferentes geometrias e $r a=40$.
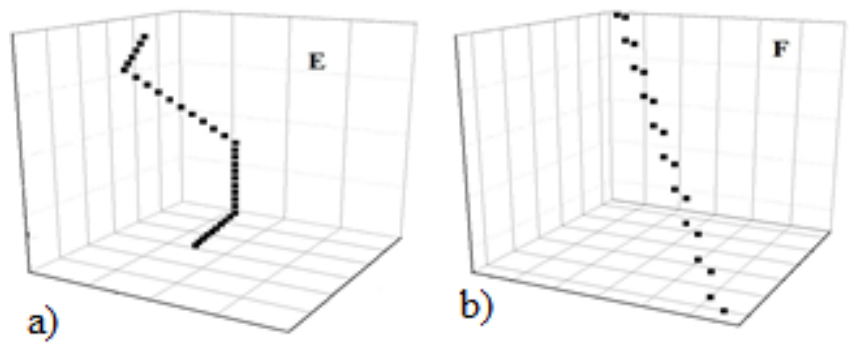

A Figura 4 mostra a simulação do valor de $\mathrm{p}_{\mathrm{c}}$, pela análise da curva de fração volumétrica de NTC em função da PC, onde o traçado na cor preta mostra o valor de $50 \%$ de percolação para 100 matrizes simuladas. A análise evidencia um resultado condizente com a simulação em 2D, onde a tortuosidade levou a diminuição do valor de $\mathrm{p}_{\mathrm{c}}$, calculando valores de $\mathrm{p}_{\mathrm{c}}=0,08 \%$ vol., para a $r a=250$. Esta diminuição pode ser explicada pela ampliação da possibilidade de contato 3D das cargas de NTC com as partículas ao seu redor, favorecendo diretamente na formação estrutural da rede percolativa. Também foi possível notar a considerável diminuição de $\mathrm{p}_{\mathrm{c}}$ em relação as matrizes quadradas, mostrando uma melhor adequação ao modelo de Balberg et al. (1984), e em concordância com os valores encontrados por Chen et al. (2014), que simularam NTC num sistema 3D e obtiveram valores de $\mathrm{p}_{\mathrm{c}}$ entre 0,548 e 0,045\% para $\mathrm{ra}$ entre 100 e 1000, respectivamente. Admitindo o efeito da tortuosidade das cargas, o valor de $\mathrm{p}_{\mathrm{c}}$ com $\mathrm{ra}$ maiores que 100 obtidos nas simulações 


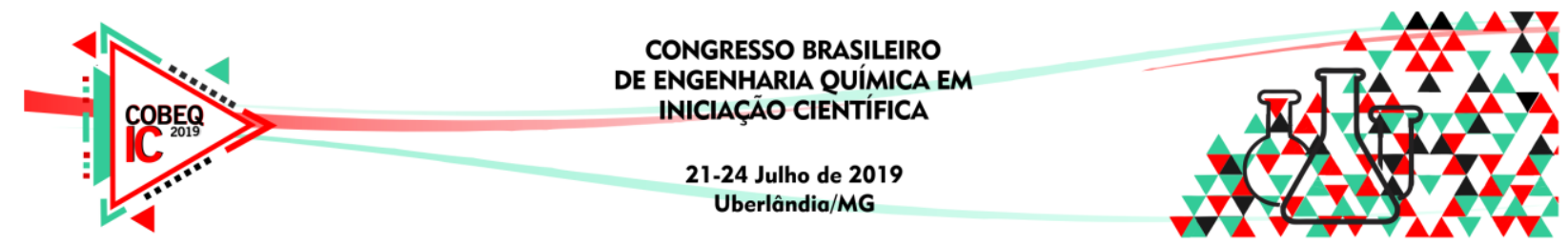

apresentaram valores próximos ao encontrado na literatura para nanocompósitos polietileno de ultra peso molecular e NTC (LISUNOVA, et al., 2007).

Figura 4 - Análise da simulação do limiar de percolação 3D pela possibilidade de conexão para: a) a geometria E; e b) geometria F.
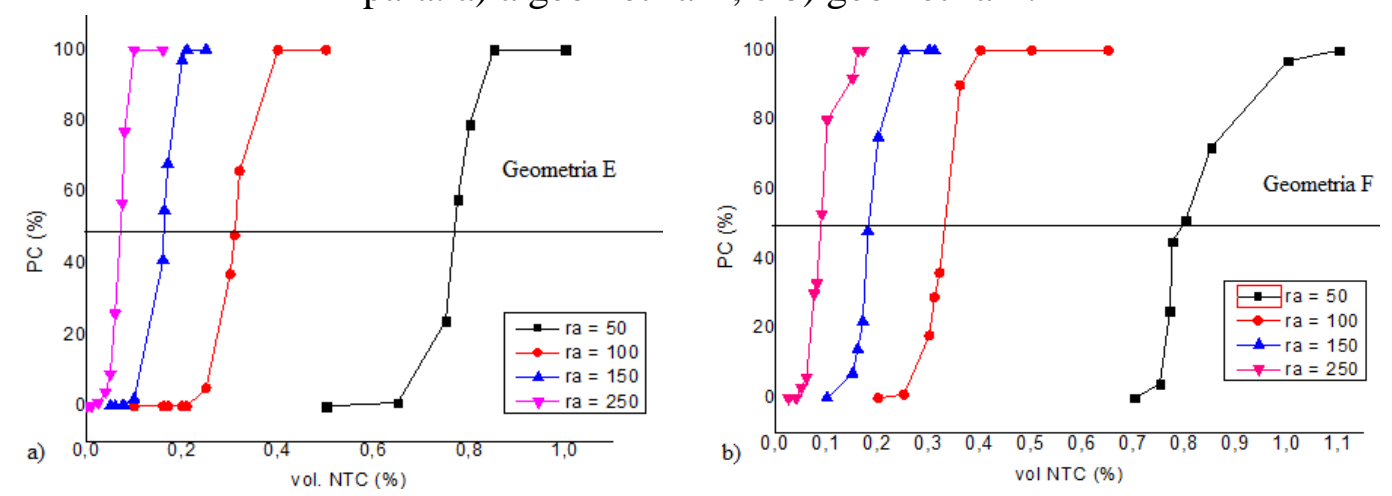

Por meio da análise do gráfico da $r a$ em função do $\mathrm{p}_{\mathrm{c}}$, Figura 5, foi realizada uma regressão não linear exponencial para o cálculo do valor de $\mathrm{p}_{\mathrm{c}}$ para os sistemas $3 \mathrm{D}$, com uma confiança de 99\%. Dessa forma, com intuito de comparar a simulação 3D com a 2D, as respectivas equações foram utilizadas para uma extrapolação do valor da $r a$, de 1000 , calculando-se um valor de percolação de $0,0754 \%$ vol., para geometria tortuosa E, e 0,0816 $\%$ vol., para a geometria F. Estes valores foram pelo menos uma ordem de grandeza inferiores aos simulados em $2 \mathrm{D}$, e mais coerentes ainda aos resultados do modelo precursor proposto por Balberg et al. (1984) que apresenta a mesma ordem de grandeza, valores próximos a $0,05 \%$ vol. para sistemas em 3D e mesma razão de aspecto.

Figura 5- Regressão não-linear exponencial $\left(\mathrm{R}^{2}=0,99\right)$ do $\mathrm{p}_{\mathrm{c}}$ das geometrias: a) E; e b) $\mathrm{F}$.
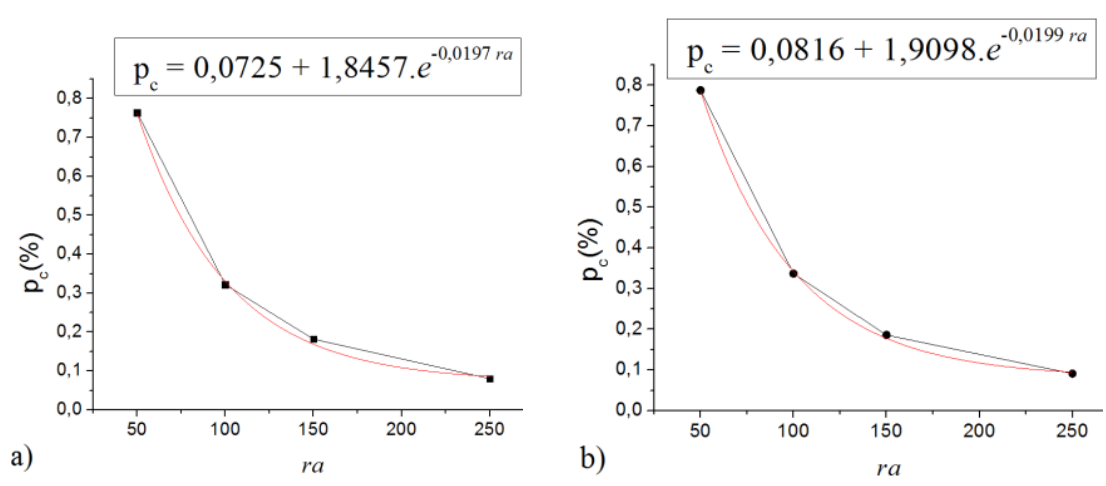

\section{CONCLUSÃO}

Através dos resultados obtidos nas simulações foi possível concluir que a geometria da carga condutora interfere no limiar de percolação, seja para sistemas $2 \mathrm{D}$ ou 3D, assim como a $r a$. Observou-se que à medida que a $r a$ do NTC aumentava, o valor de $\mathrm{p}_{\mathrm{c}}$, uma vez que as partículas apresentam uma maior dimensão fazendo com que seja necessária uma quantidade menor para formar uma rede condutora. 


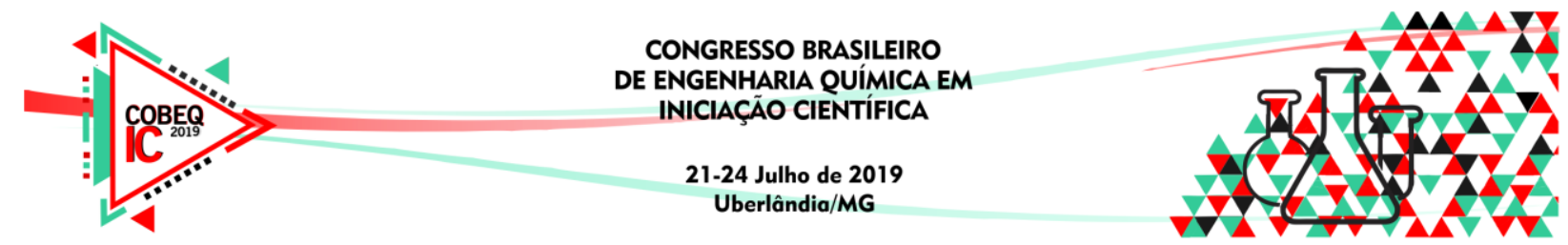

A tortuosidade das cargas influenciou diretamente no limiar, pois conforme a carga apresentava uma maior ondulação (mais tortuosa), o limiar de percolação se tornava menor, como pode ser visto nos valores obtidos para sistemas $2 \mathrm{D}$ e $3 \mathrm{D}$.

Através da representação gráfica dos resultados das simulações em 3D foi estabelecido um modelo matemático que permitiu analisar o limiar de percolação em sistemas bidimensionais para altas razões de aspectos.

\section{REFERÊNCIAS}

BALBERG, I; ANDERSON, C. H; ALEXANDER, S.; WAGNER, N. Excluded volume and its relation to the onset of percolation. Phys. Rev. B, vol.30, p. 3933,1984

BERHAN, L. SASTRY, AM. Modeling percolation in high-aspect-ratio fiber systems. II. The effect of waviness on the percolation onset. Phys. review, vol. 75, 2007.

BUG, A. L. R.; SAFRAN, S. A.;WEBMAN, I. Continuum percolation of nanocomposites with fillers of arbitrary shapes. Appl. Phys. Lett, vol.90, 2007.

CHEN, Y.; WANG, S.; PAN, F.; ZHANG, J. A numerical study on electrical percolation of polymer-matrix composites with hybrid fillers of carbon nanotubes and carbon black. Jour. of Nano., vol.2014, 2014.

COELHO, P-L.; ARMELlini, V. A. D.; MORALES, A. R. Assessment of percolation threshold simulation for individual and hybrid nanocomposites of carbon nanotubes and carbon black. Mat. Res., vol. 20 (6), p.1638-1649, 2017.

HUANG, C.; CHENG, Q. Learning from Nacre: Constructing Polymer Nanocomposites. Comp. Scien. and Tech., vol.150, p.141-166, 2017.

KUMAR, V.; RAWAL, A. Tuning the electrical percolation threshold of polymer nanocomposites with rod-like nanofillers. Polym., vol.97, p.295-299, 2016.

LISUNOVA, M.O.; MANUNYA, Y.P.; LEBOVKA, N.I.; MELEZHYK, A.V. Percolation behaviour of ultra high molecular weight polyethylene/multi-walled carbon nanotubes composites. Europ. Polym. J vol.43(3), p.949-58,2007.

LIU, Y.; KUMAR, S. Polymer/Carbon Nanotube Nano Composite Fibers-A Review. ACS Appl. Matter. Interfaces, vol.6(9), p.6069-6087, 2014.

MUNOZ-SANDOVAL, E.; CORTES-LOPEZ, A;. FLORES-GOMEZ, B.; FAJARDODIAZ, J.; SANCHEZ-SALAS, R.; LOPEZ-URIAS, F. Carbon spongetype nanostructures based on coaxial nitrogen-doped multiwalled carbon nanotubes grown by CVD using benzylamine as precursor. Carb., vol.115, p.409-421, 2017.

SOLEIMANI, H.; KHURRAM BAIG, M.; YAHYA, N.; KHODAPANAH, L.; SABET, M.; DEMIRAL, B.; BURDA, M. Impact of Carbon Nanotubes based nanofluid on Oil Recovery Efficiency using Core Flooding. Resul. in Phys., vol.9, p.39-48, 2018.

SUN, Y.; BAO, H.; GUO, Z.; YU, J. Modeling of the Electrical Percolation of Mixed Carbon Fillers in Polymer-Based Composites. Macromol., vol.42, p. 459-463, 2009. 